\title{
Co-Administration of Conjugated Linoleic Acid and Rosiglitazone Increases Atherogenic Co-Efficient and Alters Isoprenaline-Induced Vasodilatation in Rats Fed High Fat Diet
}

\author{
B. K. CHAI ${ }^{1}$, Y. S. LAU ${ }^{2}$, B. J. LOONG ${ }^{1}$, M. M. RAIS ${ }^{2}$, K. N. TING ${ }^{1}$, D. M. DHARMANI ${ }^{2}$, \\ S. K. MOHANKUMAR ${ }^{3}$
}

${ }^{1}$ Department of Biomedical Sciences, Faculty of Science, University of Nottingham Malaysia Campus, Semenyih, Selangor, Malaysia, ${ }^{2}$ Department of Pharmacology, Faculty of Medicine, University of Malaya, Kuala Lumpur, Malaysia, ${ }^{3}$ TIFAC CORE in Herbal Drugs, JSS College of Pharmacy, Udhagamandalam, JSS Academy of Higher Education and Research, Mysuru, India

Received July 4, 2017

Accepted January 5, 2018

On-line May 5, 2018

\section{Summary}

The cis(c)-9, trans(t)-11 (c9,t11) and $t 10, c 12$ isomers of conjugated linoleic acid (CLA) have been reported as agonists of peroxisome proliferator-activated receptor (PPAR) and beneficial in lipidemia and glycemia. However, it is unclear whether CLA isomers enhance or antagonize effects of conventional drugs targeting PPAR. Male Sprague-Dawley rats were fed high fat diet (HFD) for 8 weeks and treated without or with CLA, rosiglitazone or both for 4 weeks. Oral glucose tolerance and surrogate markers of insulin resistance were not significantly different for all treatments compared to untreated normal diet (ND) or HFD group, except lipoprotein levels. The combination of CLA and rosiglitazone had suppressed levels of low and high density lipoproteins ( $46 \%$ and $25 \%$, respectively), compared to HFD-alone. Conversely, the atherogenic co-efficient of the animals received HFD or HFD+rosiglitazone+CLA was 2-folds higher than ND, HFD+rosiglitazone or HFD+CLA. Isolated aortic rings from the combined CLA and rosiglitazone treated animals were less sensitive to isoprenaline-induced relaxation among endothelium-denuded aortas with a decreased efficacy and potency $\quad\left(R_{\max }=53 \pm 4.7 \%\right.$; pEC50 $\left.=6 \pm 0.2\right) \quad$ compared to endothelium-intact aortas $\left(R_{\max }=100 \pm 9.9 \%\right.$; $\left.p E C 50=7 \pm 0.2\right)$. Our findings illustrate that the combination of CLA and rosiglitazone precede the atherogenic state with impaired endotheliumindependent vasodilatation before the onset of HFD-induced insulin resistance.

\section{Key words}

PPAR agonist • High fat diet • High density lipoprotein • Rosiglitazone $\bullet$ Atherogenic

\section{Corresponding author}

S. K. Mohankumar, TIFAC CORE Herbal Drugs JSS College of Pharmacy, Udhagamandalam - 643001 , Jagadguru Sri Shivrathreeshwara University, Mysuru, India. E-mail: suresh.jsscpo@jssuni.edu.in or mssureshkum@yahoo.com

\section{Introduction}

Type 2 diabetes mellitus (T2DM) is one of the major global health burdens and have reached worldwide epidemic. T2DM is accompanied by major hallmarks such as atherogenic dyslipidemia and endothelial dysfunction (Mooradian 2009, Bakker et al. 2009). Together, these hallmarks predispose T2DM patients to cardiovascular diseases. The current options available for treatment and management of $\mathrm{T} 2 \mathrm{DM}$ are relatively limited, having problems with non-negligible side effects, and/or often require multiple combinations of drugs that mostly potentiate the adverse effects.

Thiazolidinediones (TZD) or glitazones are oral anti-hyperglycemic drugs commonly referred as "insulin sensitizers". The use of these glitazones, including rosiglitazone, have been cautioned for increased risk of cardiovascular diseases in T2DM patients (Hiatt et al. 
2013, Nissen and Wolski 2007, Nissen and Wolski 2010). This prompts the search for alternative drugs with a better clinical safety profile or suitable supplements to combat the adverse effects of TZD without altering its insulin sensitizing potential.

Conjugated linoleic acids (CLA) refer to a group of positional and geometric isomers of octadecadienoate (C18:2) with conjugated double bonds. Specifically two isomers of CLA, cis- 9 , trans-11 (c9,t11) and trans-10, cis-12 (t10,c12) isomers exist in abundance in dairy products and ruminant meats (Pariza et al. 1999) and garnered immense interest for their biological activity (Dilzer and Park 2012, Mohankumar et al. 2011, Shokryzadan et al. 2017, Taylor and Zahradka 2004). Unlike TZDs, dietary supplementation of CLA has been shown to promote weight loss in obese animals and weight gain in lean animals, prevent cardiac hypertrophy and blood pressure elevation in spontaneously hypertensive rats (Alibin et al. 2008, DeClercq et al. 2012b), and decrease LDL cholesterol in obese insulin-resistant rats and healthy human subjects (Derakhshande-Rishehri et al. 2015, Noone et al. 2002, Wanders et al. 2010). These distinct properties of CLA compared to TZDs suggest that there is a possibility that CLA may be used as an alternative supplement or substitute to combat the adverse effects of TZD.

In regards, a previous study conducted in mice fed a high fat diet demonstrated that supplementation with CLA prevented weight gain in rosiglitazone-treated mice without affecting the insulin sensitizing effects of rosiglitazone (Liu et al. 2007). However, it is not clear whether or not co-administration of CLA and TZD alter lipoproteins level and vascular functions. The present study therefore examined the effects of CLA isomers and rosiglitazone either alone or in combination on surrogate markers of insulin resistance, including lipoproteins, and on vascular contractility in rats fed high fat diet.

\section{Methods}

\section{Chemicals}

Rosiglitazone was purchased from LKT Laboratories (R5773), USA. Conjugated linoleic acid $(1: 1$ ratio of $\mathrm{c} 9, \mathrm{t} 11$ and $\mathrm{t} 10, \mathrm{c} 12)$ was purchased from $\mathrm{Nu}$ Chek Prep (UC-59-AX), USA. (R)-(-)-Phenylephrine hydrochloride and isoproterenol hydrochloride (isoprenaline) were purchased from Tocris Bioscience, UK.

\section{Animals and diet}

Thirty male Sprague-Dawley (SD) rats (6 weeks old) were received (Monash University Malaysia Campus, Malaysia) and acclimatized with normal rodent chow diet (Altromin, 1324, Germany) for two weeks. We selectively used male rats as they are known to be more susceptible to metabolic disorder than female rats and to avoid the estrous cycle mediated interference on metabolic parameters in female rats. The experimental protocol was approved by the Faculty of Medicine Institutional Animal Care and Use Committee, University of Malaya (Approval number: 2014-03-05/PHAR/R/ DDM) and the Animal Welfare and Ethical Review Body, University of Nottingham. Food and water were provided ad libitum.

The rats were divided into groups fed with normal diet (ND; $4.10 \%$ total energy from fat; 6 rats) and high fat diet (24 rats; $60 \%$ total energy from fat; Research Diets, D12492, USA). After completion of 8 weeks of HFD feeding, rats were divided into the following groups and treated for 4 weeks: HFD control (HFD), HFD treated with rosiglitazone (HFD+RGZ), HFD treated with CLA (HFD+CLA) and HFD treated with rosiglitazone and CLA (HFD+RGZ+CLA). CLA was dissolved in $5 \% \mathrm{w} / \mathrm{w}$ fat free milk and administered at $0.2 \mathrm{~g}$ per animal per day by oral gavage. The dose of CLA is based on the human consumption equivalent, which amounts to $1-2 \%$ of dietary weight per day. On average, a rat consumes $20 \mathrm{~g}$ of food per day. With this assumption, we calculate that each rat should be given $0.2 \mathrm{~g}$ of CLA per day. The control rats received only $5 \% \mathrm{w} / \mathrm{w}$ fat free milk. Rosiglitazone was administered daily by intraperitoneal injection at $2 \mathrm{mg} / \mathrm{kg}$ body weight and control rats received only saline injection. The dose of rosiglitazone is based on the previously published study (Zhao et al. 2012).

Body weight, feed intake and blood glucose (Accu-Check blood glucose monitor) were recorded weekly, except terminal blood glucose. A week before the sacrifice, rats were fasted overnight and undergone oral glucose tolerance test (OGTT) as previously described (Noto et al. 2006). At the end of the study, all rats were fasted overnight, asphyxiated with $\mathrm{CO}_{2}$ and their blood and organs were collected. The blood samples were centrifuged at 2,000 rpm for $10 \mathrm{~min}$ and the serum separated were stored at $-80^{\circ} \mathrm{C}$ until further analyses. The thoracic aortas were isolated for vascular contractility studies. 


\section{Serum biochemical analysis}

Terminal fasting blood samples were analyzed for glucose (Cayman, 10009582, Ann Arbor, MI, USA), total cholesterol (Cayman, 10007640, USA), total triglycerides (Zen-Bio, STG-1-NC, USA), high density lipoprotein (HDL) and LDL/vLDL (BioVision, K613-100, Milpitas, CA, USA) and insulin levels (Alpco Diagnostics, 80-INSRT-E01, Salem, NH, USA) according to manufacturer's instructions. The colorimetric and fluorimetric end points were measured in Varioskan Flash Multimode Reader (Thermo Scientific, USA).

\section{In vitro organ bath experiments}

This procedure was performed as previously described (Loong et al. 2015). Briefly, thoracic aortae were immediately excised and transferred into cold Krebs-Ringer bicarbonate solution upon sacrifice. In an aerated tissue bath, the aortic rings $(4 \mathrm{~mm})$ were left in the bath for $30 \mathrm{~min}$ to equilibrate before the application of $2 \mathrm{~g}$ weight tension. Endothelium denuded rings were prepared by gently rubbing the intimal surface using the blunt tip of stainless steel forceps. Successful denudation was confirmed if the tissue produced less than $30 \%$ relaxation in the presence of $1 \times 10^{-6} \mathrm{M}$ carbachol in the pre-constricted rings. All aortic rings were pre-contracted to at least $70 \%$ of maximal contraction with $0.1 \mu \mathrm{M}$ phenylephrine before the addition of isoprenaline $\left(1 \times 10^{-10}-3 \times 10^{-5} \mathrm{M}\right)$. Maximal relaxation $\left(\mathrm{R}_{\max }\right)$ and pEC50 values were calculated using Prism version 6.0 (GraphPad Software, USA) in which $\mathrm{EC}_{50}$ is the concentration of isoprenaline that produces $50 \%$ of maximal relaxation and $\mathrm{pEC}_{50}$ is taken as the negative common logarithm of $\mathrm{EC}_{50}$.

\section{Calculations}

Weekly energy intake was calculated with the following formula:

$$
\text { Energy intake }\left(K J g^{-1}\right)=\frac{\text { Metabolisable energy per gram of food }\left(K J g^{-1}\right) \times \text { Average food consumed per rat }(g)}{\text { Average body weight per rat }(g)}
$$

Homeostatic model assessment-insulin resistance (HOMA-IR) was calculated by taking the product of blood glucose in $\mathrm{mg} / \mathrm{dl}$ and serum insulin in $\mathrm{mU} / \mathrm{l}$, divided by 405 , as described previously (Mohankumar et al. 2013).

Lipoprotein ratios were obtained by using serum total, LDL and HDL cholesterol readings in mmol/l. Non-HDL cholesterol was calculated by subtracting HDL-cholesterol from total cholesterol (Martirosyan et al. 2007). Ratios of LDL:HDL, total cholesterol (TC):HDL and non-HDL:HDL were obtained.

\section{Statistics}

Results are shown as means \pm standard error of the mean for the number of rats tested. Statistical analyses were performed using unpaired Student's t-test to compare two groups and one-way ANOVA with Tukey's post hoc tests to compare three or more groups. Repeated measure one-way ANOVA was performed for concentration-responses and time responses. Analyses were done on SPSS version 22 (IBM, USA) and graphics prepared using Prism version 6.0 (GraphPad Software, USA). A probability of less than $0.05(\mathrm{p}<0.05)$ was considered to be statistically significant. Diet specific differences were compared with $\mathrm{ND}$ and treatment specific differences were compared with HFD.

\section{Results}

\section{Physical characteristics and energy intake}

First, we analyzed the effect of diet and treatment on body weight, organ weight and food intake. From week 4 onwards, all groups fed high fat diet (HFD, HFD+RGZ, HFD+CLA, HFD+RGZ+CLA) were $20 \%$ heavier than normal diet (ND) (Fig. 1A). Organ weights were similar in all groups except fat pads including epididymal, perirenal and mesenteric fats, the weight of groups fed high fat diet were 1.3 to 2.7 folds higher than normal diet (Table 1). However, no treatment specific difference was observed $(\mathrm{p}>0.05)$. Energy intakes, in kilojoules measured weekly, were similar across all groups (Fig. 1B).

\section{Blood glucose and insulin}

Since hyperglycemia and insulin resistance are major hallmarks of T2DM and metabolic syndrome (Kaur 2014), we measured the fasting blood glucose and insulin levels to determine the effect of the diet and/or treatment of glycemia.

The fasting blood glucose levels were found to be similar across all groups (Fig. 2A). All rats were found to have similar glucose clearance with respect to OGTT (Fig. 2B). In addition, whilst rats belonging to 
HFD+RGZ, HFD+CLA and HFD+CLA+RGZ groups geared towards improved insulin sensitivity as shown by homeostatic model assessment of insulin resistance
(HOMA-IR) index (Fig. 2C), they were not statistically different from ND or HFD control groups.

Table 1. Effects of diet and treatment on organ weights.

\begin{tabular}{|c|c|c|c|c|c|}
\hline & ND & HFD & HFD+RGZ & HFD+CLA & HFD+RGZ+CLA \\
\hline Heart $(g)$ & $1.28 \pm 0.07$ & $1.47 \pm 0.05$ & $1.52 \pm 0.10$ & $1.51 \pm 0.04$ & $1.43 \pm 0.04$ \\
\hline Liver $(g)$ & $10.67 \pm 0.06$ & $10.33 \pm 0.46$ & $11.00 \pm 0.11$ & $11.19 \pm 0.35$ & $10.00 \pm 0.66$ \\
\hline \multicolumn{6}{|l|}{ Fat pads } \\
\hline Epididymal fat (g) & $4.24 \pm 0.45$ & $8.15 \pm 0.96^{*}$ & $7.90 \pm 0.14^{*}$ & $9.06 \pm 0.99 *$ & $7.50 \pm 0.97^{*}$ \\
\hline Perirenal fat ( $g$ ) & $3.8 \pm 0.46$ & $8.64 \pm 1.4^{*}$ & $9.81 \pm 1.16^{*}$ & $10.54 \pm 0.89^{*}$ & $8.87 \pm 1.20^{*}$ \\
\hline Mesenteric fat ( $g$ ) & $2.14 \pm 0.26$ & $3.39 \pm 0.45^{*}$ & $3.78 \pm 0.32 *$ & $3.60 \pm 0.23^{*}$ & $2.92 \pm 0.48^{*}$ \\
\hline Pancreas & $1.03 \pm 0.14$ & $1.45 \pm 0.18$ & $1.30 \pm 0.18$ & $1.61 \pm 0.15$ & $1.50 \pm 0.18$ \\
\hline \multicolumn{6}{|l|}{ Kidneys } \\
\hline Right kidney (g) & $1.46 \pm 0.11$ & $1.54 \pm 0.08$ & $1.62 \pm 0.13$ & $1.69 \pm 0.08$ & $1.60 \pm 0.06$ \\
\hline Left kidney (g) & $1.44 \pm 0.11$ & $1.50 \pm 0.08$ & $1.56 \pm 0.14$ & $1.67 \pm 0.04$ & $1.57 \pm 0.08$ \\
\hline Gastrocnemius muscle (g) & $2.23 \pm 0.36$ & $2.33 \pm 0.18$ & $3.00 \pm 0.50$ & $2.21 \pm 0.10$ & $2.16 \pm 0.16$ \\
\hline Soleus muscle (g) & $0.16 \pm 0.01$ & $0.20 \pm 0.02$ & $0.19 \pm 0.01$ & $0.20 \pm 0.07$ & $0.20 \pm 0.02$ \\
\hline
\end{tabular}

Data were presented as mean $\pm \mathrm{SEM}, \mathrm{n}=6$ for each group. $* \mathrm{p}<0.05$ compared to ND.

A

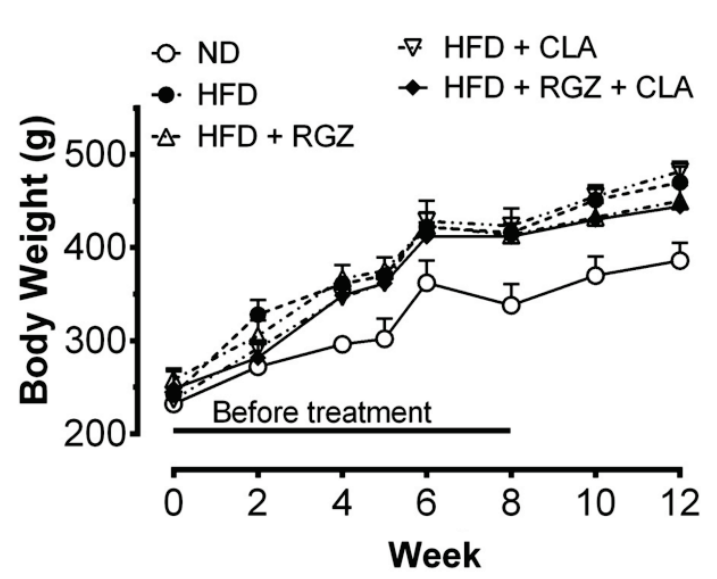

B

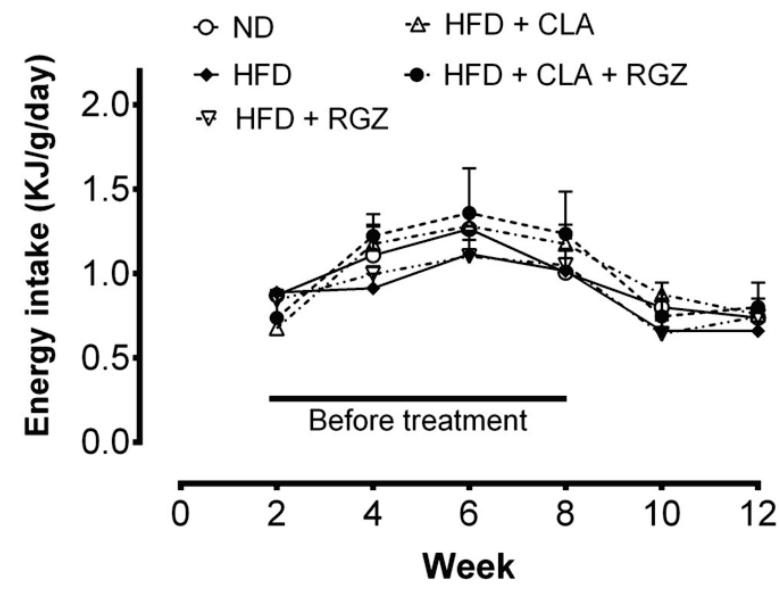

Fig. 1. Effect of diet and treatments on body weight and energy intake. (A) Body weight of Sprague-Dawley rats throughout the study. (B) Average energy intake of Sprague-Dawley rats throughout the study. Data is presented as mean \pm SEM, $n=6$ in all groups. ${ }^{\#} p<0.05$ compared to ND.

\section{Serum lipoprotein levels and ratios}

Atherogenic dyslipidemia is one of the critical metabolic characteristic of T2DM (Mooradian 2009), hence various serum lipid markers of dyslipidemia were measured. As depicted in Figures 3A and 3D, neither diet nor treatments have altered the serum TC and triglyceride levels. The serum LDL level was $46 \%$ reduced in HFD + CLA + RGZ group in comparison to HFD alone $(p<0.05$, Fig. 3B). Conversely, animals from HFD,
HFD+RGZ and HFD+CLA have shown 1.3 folds elevated levels of HDL compared to ND $(p<0.05)$, whereas HFD+RGZ+CLA have $25 \%$ suppressed serum HDL than HFD group and the level was similar to the level measured in ND group (Fig. 3C). A plethora of published studies reported that cholesterol ratios, especially LDL:HDL, TC:HDL and non-HDL:HDL ratios have been recognized as the critical markers of atherogenicity and are widely used to assess 
cardiovascular risk (van Deventer et al. 2011, Hermans et al. 2007, Lu et al. 2003). As shown in Figures 4A and 4B, the TC:HDL and non-HDL:HDL ratios in the animals received HFD or $\mathrm{HFD}+\mathrm{RGZ}+\mathrm{CLA}$ was 2-folds higher than ND $(p<0.05)$, whereas no significant changes observed for HFD+RGZ and HFD+CLA. Although the LDL:HDL ratio was lower in treatment groups (Fig. 4C), they were not significantly different compared to ND and HFD, respectively ( $p>0.05)$.
A

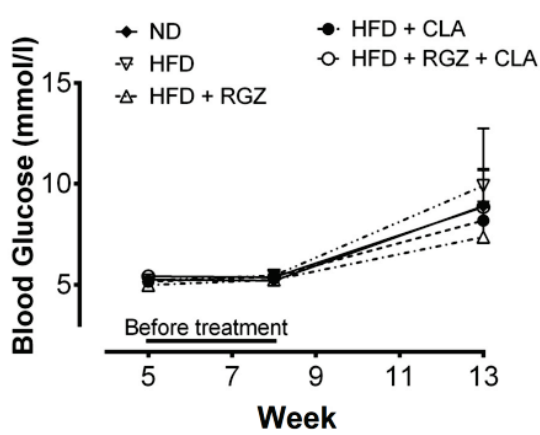

C

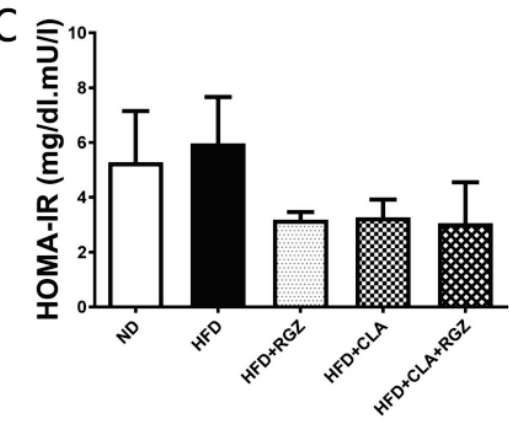

B

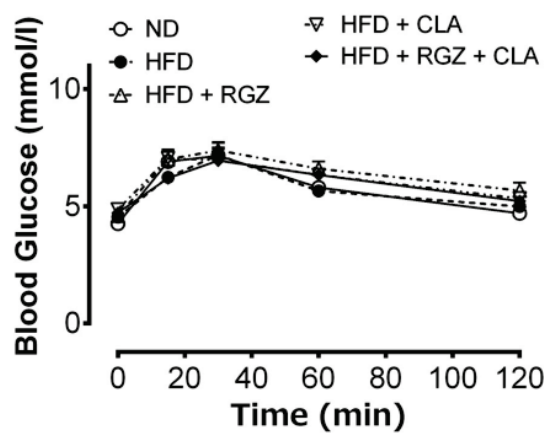

Fig. 2. Effect of diet and treatment on glycemia (A) Measurement of blood glucose level. Blood glucose on week 5 and week 8 measured using tail prick method; terminal blood glucose on week 13 measured using glucose assay kit. Data is presented as mean \pm SEM, $n=6$ in all groups. (B) Oral glucose tolerance test (OGTT). A day before the 12 week, rats were fasted overnight before undergoing oral glucose tolerance test. (C) HOMA-IR of rats at termination. Data were presented as mean \pm SEM, $n=6$ in all groups.

Fig. 3. Effect of diet and treatments on lipid profiles. (A) Total cholesterol. (B) LDL-cholesterol. (C) HDL-cholesterol. (D) Triglycerides. Data were presented as mean \pm SEM, $n=6$ in all groups. ${ }^{*} p<0.05$ compared to ND. ${ }^{*} p<0.05$ compared to HFD. 
Isoprenaline-induced vasorelaxation

A set of preliminary experiments were conducted to optimize the experimental conditions for isoprenaline-induced relaxation (data not shown). The degree of initial contraction induced by $0.1 \mu \mathrm{M}$ phenylephrine for both control and treatment groups either without or with endothelium were not statistically significant ( $\mathrm{p}>0.05$; data not shown). A representative trace of isoprenaline-induced relaxation in aortic rings excised from $\mathrm{SD}$ rats fed normal rodent chow diet is shown in Figure 5A.
A

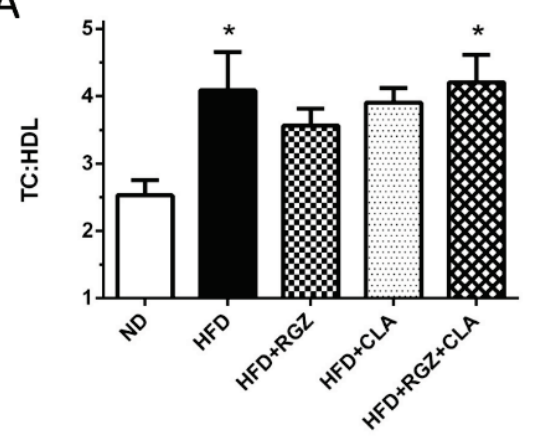

B

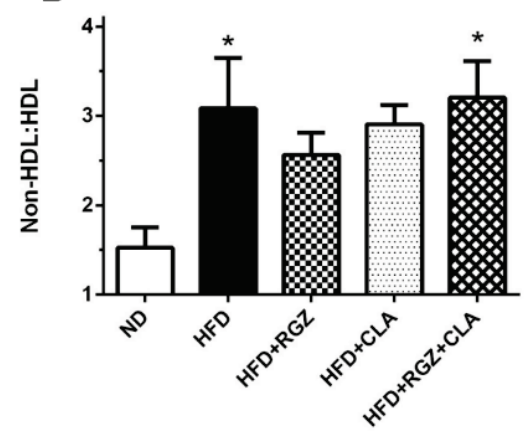

Fig. 4. Effect of diet and treatments on lipoprotein ratios. (A) TC:HDL ratio. (B) non-HDL:HDL ratio. (C) LDL:HDL ratio. Data were presented as mean $\pm S E M, n=6$ for each group. $* p<0.05$ compared to ND.

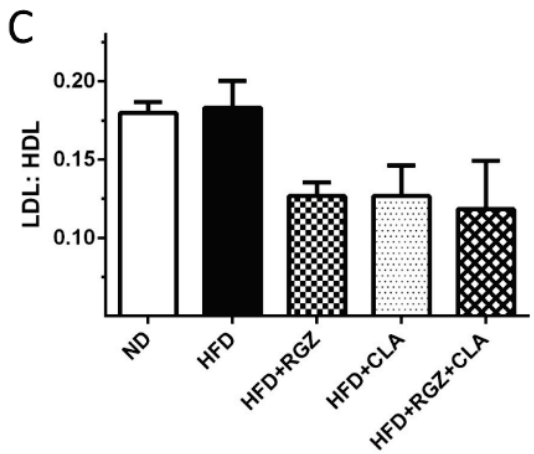

The effect of diet and treatment on isoprenalineinduced vascular relaxation in isolated aortic rings excised from the rats was examined. The endothelial layer was removed on some of the aortic rings to check if the endothelium has been affected by the various treatments. All isolated aortic rings exhibited concentration-dependent vasodilatation in response to isoprenaline as the vasodilator. Repeated measures analysis indicated that the isoprenaline-induced vasorelaxation was significantly different between ND, HFD and HFD + RGZ+CLA regardless of endothelial presence (Figs 5B and 5C).

Among endothelium intact rings (Fig. 5B), all rings fed HFD, regardless of treatments were more responsive than $\mathrm{ND}$. $\mathrm{HFD}+\mathrm{RGZ}+\mathrm{CLA}$ was the most responsive to isoprenaline. This was followed by HFD. The responsiveness of HFD+RGZ is intermediate of HFD and HFD + RGZ+CLA, while HFD+CLA is intermediate of HFD and ND. All groups exhibited similar pEC50 and $\mathrm{R}_{\max }$ (Table 2 and Fig. 5B). However, the trend was reversed in endothelium denuded rings (Table 2 and Fig. 5C). Removal of endothelium worsened the isoprenaline-induced vasorelaxation in all HFD and treatment groups. The relaxation was severely affected in aortic rings obtained from HFD+RGZ+CLA treated animals ( $\mathrm{pEC} 50=6 \pm 0.2, \mathrm{R}_{\max }=53.6 \pm 4.7 \%$ ) compared to $\mathrm{ND}$ ( $\mathrm{pEC} 50=7 \pm 0.2, \mathrm{R}_{\max }=113 \pm 22 \%$ ). Likewise, aortic rings from both HFD and HFD+CLA had diminished response to isoprenaline compared to $\mathrm{ND}$ (pEC50 $=6.6 \pm 0.1, \mathrm{R}_{\max }=93 \pm 24.2 \%$ and $\mathrm{pEC} 50=6.4 \pm 0.4$, $\mathrm{R}_{\max }=77.70 \pm 22.8 \%$, respectively). Notably, the efficacy and potency of isoprenaline was significantly reduced in endothelium denuded aortic rings in comparison to endothelium intact aortic rings of $\mathrm{HFD}+\mathrm{RGZ}+\mathrm{CLA}$ treated rats (Endothelium intact: $\mathrm{pEC} 50=7 \pm 0.2$, $\mathrm{R}_{\max }=100 \pm 9.9 \% \quad$ vs. endothelium denuded: pEC50 $=6 \pm 0.2, \quad R_{\max }=53 \pm 4.7 \%$ ) (Figs 5B and 5C), indicating the critical role of endothelium in protecting the PPAR agonists-mediated atherogenic risks.

\section{Discussion}

Our key findings of this study were that the co-administration of CLA and rosiglitazone to SD rats 
fed HFD (60\% energy from fat) had elevated TC:HDL and non HDL:HDL ratio, suppressed LDL and HDL level, and a marked decrease in endotheliumindependent aortic relaxation, suggesting that CLA and rosiglitazone precede the atherogenic state and impaired endothelium-independent vasodilatation despite the reduction of HFD-induced insulin resistance.

There have been previous efforts to examine feeding rosiglitazone and CLA alone in rodent models fed with HFD. Dietary supplementation with CLA for seven weeks, regardless of isomer composition, led to an improvement in glucose tolerance, insulin resistance and serum triglycerides (Choi et al. 2004). Likewise, treatment with rosiglitazone improved insulin sensitivity with a side effect of increasing serum LDL (Zhao et al.
2012). It is noteworthy that in this study SD rats were treated for four weeks to induce acute, instead of chronic effects as showed previously (Lessard et al. 2007, Murthy et al. 2005, Umrani et al. 2002). The onset of insulin resistance in animals can be influenced by a number of factors such as feeding period, type of fat in the diet and genotype of animals. We observed neither change in glucose tolerance nor obvious insulin resistance following 12 weeks of HFD. We reason that the rats used in our study possibly mimic the obesity resistant phenotype, since we did not observe significant impairments in glucose tolerance following HFD supplementation for 12 weeks, whilst rats gained marginal rise in body and fat pad weights compared to their normal diet counterparts (Dake and Oltman 2015).
A
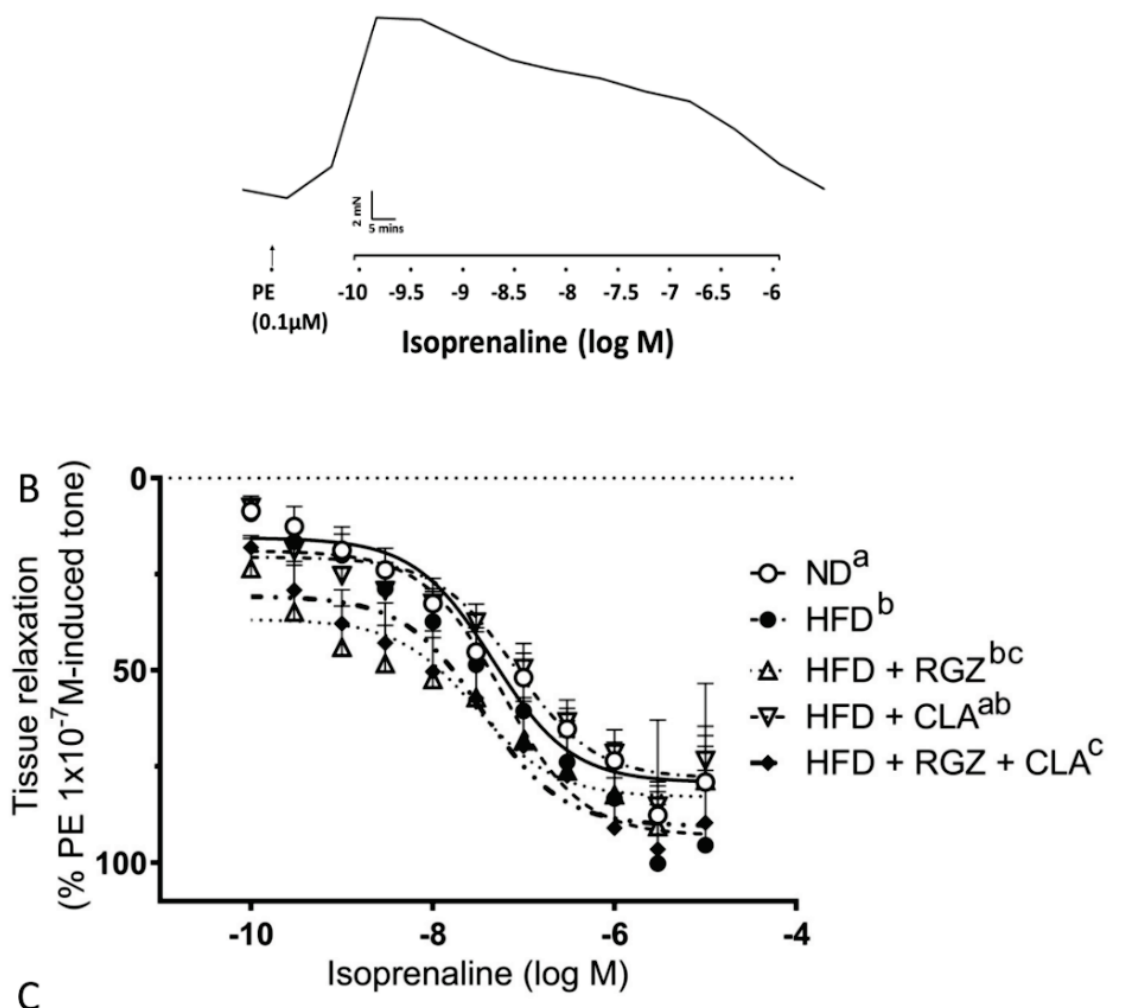

C

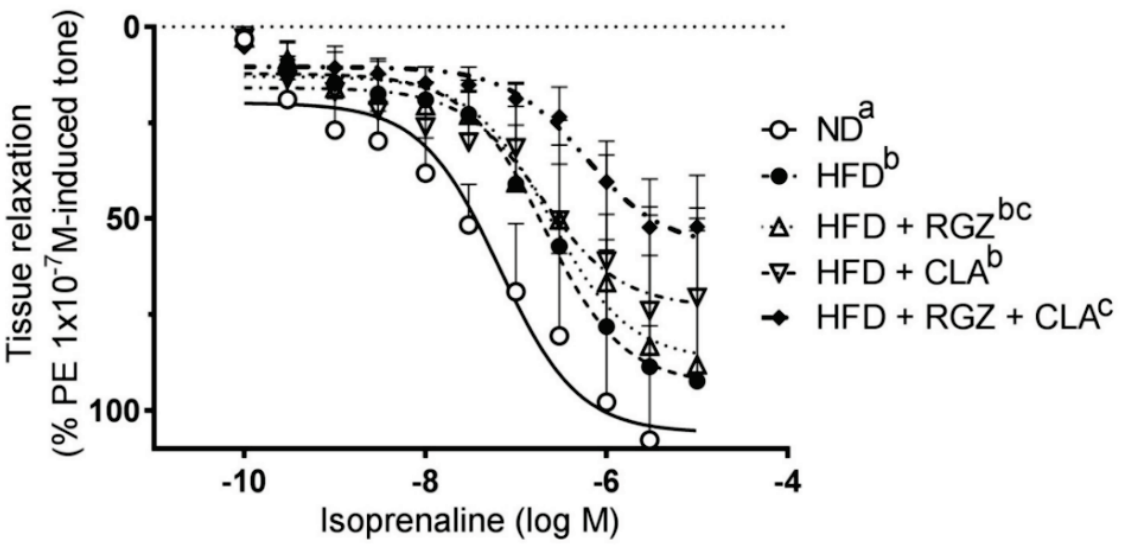

Fig. 5. Effect of diet and treatments on isoprenaline-induced aortic ring relaxation. (A) Representative trace of isoprenaline-induced aortic ring relaxation of normal SD rats. (B) Relaxation of endothelium-intact aortic rings. (C) Relaxation of endothelium-denuded aortic rings. Data were presented as mean $\pm \mathrm{SEM}$, $n=4-6$ for endothelium intact and $n=3$ for endothelium denuded. For $B$ and $C$, statistical significance is indicated by letters next to the legends, groups having the same letter(s) are not significant from each other. 
Table 2. pEC50 and maximal relaxation $\left(R_{\max }\right)$ of aortic rings isolated from rats.

\begin{tabular}{lccccc}
\hline & ND & HFD & HFD+RGZ & HFD+CLA & HFD+RGZ+CLA \\
\hline$p E C 50$ E & $7.414 \pm 0.236$ & $7.271 \pm 0.239$ & $7.979 \pm 0.453$ & $7.128 \pm 0.2414$ & $7.453 \pm 0.2088$ \\
$p E C 50$ - & $7.105 \pm 0.176$ & $6.600 \pm 0.127$ & $6.583 \pm 0.375^{*}$ & $6.398 \pm 0.3754$ & $6.133 \pm 0.231^{*}$ \\
$R_{\max } E+(\%$ PE induced tone) & $89.75 \pm 6.95$ & $102.50 \pm 13.18$ & $91.00 \pm 12.02$ & $85.93 \pm 6.16$ & $100.2 \pm 9.92$ \\
$R_{\max } E-(\%$ PE induced tone) & $113.4 \pm 22.45$ & $93.19 \pm 24.15$ & $90.60 \pm 29.71$ & $77.70 \pm 22.81$ & $53.58 \pm 4.68^{*}$ \\
\hline
\end{tabular}

Data were presented as mean \pm SEM. * $p<0.05$ compared to $E+$.

Alternatively, as observed in earlier studies (Pranprawit et al. 2013, Stark et al. 2000, Xu et al. 2010), the period of less than 12 weeks is too short to induce metabolic changes in obesity resistant rats. In contrast, Huang et al. (2004) reported reduction in glucose tolerance after 8 weeks of high fat diet feeding. It is apparent that no standardization of diet-induced obesity exists in literature despite using the common strain of SD rats. In a study that report the body weight reduction in SD rats with dietary supplementation of CLA, the animals have been fed with CLA from the beginning of the study together with HFD; moreover CLA elicits isomer-specific effects (Choi et al. 2004). Conversely, in another study dietary supplementation of CLA mixture for 6 weeks in rats showed no change in body weight (Moya-Camarena et al. 1999). Thus, our observation that the absence of weight reduction following oral administration of CLA mixture $(0.2 \mathrm{~g} /$ day $)$ for 4 weeks in SD rats pre fed with HFD for 8 weeks could be explained by the duration of CLA supplementation and isomer composition of CLA mixture.

Dyslipidemia is linked to vascular dysfunction in T2DM (Dixon et al. 1999). Generally, increased circulating concentration of HDL is associated with lowered risk of cardiovascular disease. HDL are lipoprotein particles that mediate reverse cholesterol transport (RCT) with apolipoprotein $\mathrm{A}$ as the major protein component. Beyond its role in RCT, HDL binds to scavenger receptor (SR)-B1 and SR-B2 and directly exerts vasoprotective effects on the endothelium (Calabresi 2003). As demonstrated previously by Hayek et al. (1993), the increase in HDL is typical in rodents fed HFD. Thus, the HDL rise in our study is possibly an adaptive response to maintain vascular tone to compensate the increased atherogenicity brought by HFD.

CLA are known to be weak PPAR $\alpha$ agonists (Moya-Camarena et al. 1999). While studies of rosiglitazone and CLA are difficult to come by, our observation of $\mathrm{HDL}$ reduction in $\mathrm{HFD}+\mathrm{CLA}+\mathrm{RGZ}$ treated rats is in agreement with the previous observations in case reports ( $\mathrm{Im}$ et al. 2010, Normen et al. 2004, Schwing et al. 2010) and a clinical trial (Linz et al. 2014) in which rosiglitazone and fenofibrate, a commonly prescribed PPAR $\alpha$ agonist, were simultaneously administered to human patients. Whilst the biological actions of CLA on HDL and insulin sensitivity were complicated by isomer and composition dependence, our observation that a 1:1 mixture of $\mathrm{c} 9, \mathrm{t} 11$ and t10,c12-CLA increases HDL level is similar to a recent published study conducted in Wistar rats fed HFD (de Almeida et al. 2015). We have noted that HFD+RGZ+CLA brought about suppression of LDL and HDL without significant reduction in LDL:HDL ratio. Since fractions outside LDL and HDL are also involved in atherogenesis, we make use of the non-HDL:HDL ratio, also known as the atherogenic coefficient, that has been proposed to be better predictors of cardiovascular risks (Hermans et al. 2007, Kim et al. 2013). Another ratio, TC:HDL, is linked to non-HDL:HDL by a linear relationship. Both ratios were increased in HFD+RGZ+CLA group despite having similar HDL level with ND suggesting increased atherogenicity, that is corroborated with the decreased ability of aortic rings to respond to isoprenaline-mediated vasodilatation in HFD+RGZ+CLA treatment group.

It is well established that the changes in vascular function when fed an atherogenic diet precede the onset of insulin resistance and cardiovascular diseases. In agreement with these studies (Garcia-Prieto et al. 2015, Stark et al. 2000), we observed diminished endotheliumindependent vasodilatation without significant changes in glycemia. The vascular endothelium forms the inner lining of blood vessels and plays important role in maintaining vessel physiology (Deanfield et al. 2007, Hadi et al. 2005). Under physiological conditions, blood vessels are kept quiescent and dilated by endothelial secretion such as nitric oxide (NO). However, endothelial denudation due to apoptotic loss of endothelium is known 
to occur under the diabetic and insulin resistant state (Avogaro et al. 2011, Sena et al. 2013). The increased responsiveness in HFD-fed endothelium intact rings may be an acute adaptive response to high circulating lipoproteins. Similar observations were recorded in obese spontaneously hypertensive rats (Kagota et al. 2004).

Isoprenaline-induced vascular relaxation is dependent on $\beta$-adrenergic receptors present on both endothelium and vascular smooth muscle (VSM) (Akimoto et al. 2002). Endothelium independent relaxation of VSM is mediated by NO produced in situ (Bernhardt et al. 1991). Our observation of vascular impairment in rosiglitazone fed endothelium denuded rings agrees with a previous finding, which stemmed from the improvement in endothelial production of $\mathrm{NO}$ but impaired response of VSM in rats (Onuta et al. 2008). The decreased responsiveness of endothelium denuded rings can be explained by rosiglitazone, via PPAR $\gamma$, induced upregulation of CD36 in the VSM (Ballesteros et al. 2014, Kleinhenz et al. 2015), as CD36 is responsive to LDL and lead to inhibition of cGMP synthesis in the VSM (Isenberg et al. 2009). Conversely, it has been reported that $\mathrm{t} 10, \mathrm{c} 12-\mathrm{CLA}$ increases NO formation in human endothelium (DeClercq et al. 2012a), nonetheless our observations warrant further studies to elucidate the precise mechanisms by which CLA modulate endothelium-independent relaxation.

The ability of aorta to constrict in obesity has been shown to be impaired due to many factors, including overnutrition of dietary fats (Stapleton et al. 2008). It has been previously reported that under certain environmental stressors, including HFD, the VSM cells tends to lose its contractile ability due to the remodeling of VSM into synthetic phenotype (Beamish et al. 2010). Watts et al. (2012) have demonstrated the impairments in phenylephrine-mediated contraction in aorta excised from
SD rats fed HFD for 17 weeks. Conversely, we observed no differences with phenylephrine-induced contraction in both control and HFD diet groups. Whilst we reason that the differences could be due to the duration of HFD feeding, proportion of HFD and age of rats, the impairment in contractile ability of VSM in rodents fed HFD was shown to be more prominent in small mesenteric arteries than the thoracic aorta (Soares et al. 2017).

In summary, while glucose tolerance and HOMAIR may be unchanged, simultaneous administration of CLA and rosiglitazone led to increase in atherogenic coefficient. The increase in atherogenicity is coupled with vascular resistance in the context of endothelial loss. This observation hints the complex interplay between nutrientdrug interactions and warrants further investigation on their molecular mechanisms. Whilst it is believed that the use of normal rodent phenotype is limited in studying lipoprotein metabolism due to higher level of circulating HDL compared to humans, our observations, the linkage of cholesterol ratios and vascular resistance in SD rats fed HFD provides a reasonable basis for revisiting the lipoprotein dynamics in rodents.

\section{Conflict of Interest}

There is no conflict of interest.

\section{Acknowledgements}

We thank Ms. Ling Wei Chih for her help with animal care. We thank Ms. Akila Raghavan for analyzing blood glucose and total cholesterol. We would like to acknowledge the generous infrastructure provided by University of Nottingham Malaysia Campus (UNMC) and University of Malaya. This research was supported by UNMC Pump Priming grant and the Fundamental Research Grant Scheme from the Ministry of Higher Education, Malaysia (grant number: 64924).

\section{References}

AKIMOTO Y, HORINOUCHI T, SHIBANO M, MATSUSHITA M, YAMASHITA Y, OKAMOTO T, YAMAKI F, TANAKA Y, KOIKE K: Nitric oxide (NO) primarily accounts for endothelium-dependent component of betaadrenoceptor-activated smooth muscle relaxation of mouse aorta in response to isoprenaline. J Smooth Muscle Res 38: 87-99, 2002.

ALIBIN CP, KOPILAS MA, ANDERSON HD: Suppression of cardiac myocyte hypertrophy by conjugated linoleic acid: role of peroxisome proliferator-activated receptors alpha and gamma. J Biol Chem 283: 10707-10715, 2008.

AVOGARO A, ALBIERO M, MENEGAZZO L, DE KREUTZENBERG S, FADINI GP: Endothelial dysfunction in diabetes: The role of reparatory mechanisms. Diabetes Care 34 (Suppl 2): S285-S290, 2011.

BAKKER W, ERINGA EC, SIPKEMA P, VAN HINSBERGH VW: Endothelial dysfunction and diabetes: roles of hyperglycemia, impaired insulin signaling and obesity. Cell Tissue Res 335: 165-189, 2009. 
BALLESTEROS I, CUARTERO MI, PRADILLO JM, DE LA PARRA J, PÉREZ-RUIZ A, CORBÍ A, RICOTE M, HAMILTON J A, SOBRADO M, VIVANCOS J, NOMBELA F, LIZASOAIN I, MORO MA: Rosiglitazoneinduced CD36 up-regulation resolves inflammation by PPAR $\gamma$ and 5-LO-dependent pathways. J Leukoc Biol 95: 587-598, 2014.

BEAMISH JA, HE P, KOTTKE-MARCHANT K, MARCHANT RE: Molecular regulation of contractile smooth muscle cell phenotype: implications for vascular tissue engineering. Tissue Eng Part B Rev 16: 467-491, 2010.

BERNHARDT J, TSCHUDI MR, DOHI Y, GUT I, URWYLER B, BÜHLER FR, LÜSCHER TF: Release of nitric oxide from human vascular smooth muscle cells. Biochem Biophys Res Commun 180: 907-912, 1991.

CALABRESI L: Endothelial protection by high-density lipoproteins: from bench to bedside. Arterioscler Thromb Vasc Biol 23: 1724-1731, 2003.

CHOI JS, JUNG MH, PARK HS, SONG J: Effect of conjugated linoleic acid isomers on insulin resistance and mRNA levels of genes regulating energy metabolism in high-fat-fed rats. Nutrition 20: 1008-1017, 2004.

DAKE BL, OLTMAN CL: Cardiovascular, metabolic, and coronary dysfunction in high-fat-fed obesity-resistant/prone rats. Obesity 23: 623-629, 2015.

DE ALMEIDA MM, DE SOUZA YO, DUTRA LUQUETTI SC, SABARENSE CM, Do AMARAL CORREA JO, DA CONCEIÇÃO EP, LISBOA PC, DE MOURA EG, ANDRADE SOARES SM, MOURA GUALBERTO AC, GAMEIRO J, DA GAMA MA, FERRAZ LOPES FC, GONZÁLEZ GARCIA RM: Cis-9, trans-11 and trans-10, cis-12 CLA mixture does not change body composition, induces insulin resistance and increases serum HDL cholesterol level in rats. J Oleo Sci 64: 539-551, 2015.

DEANFIELD JE, HALCOX JP, RABELINK TJ: Endothelial function and dysfunction: testing and clinical relevance. Circulation 115: 1285-1295, 2007.

DeCLERCQ V, TAYLOR CG, WIGLE J, WRIGHT B, TWOREK L, ZAHRADKA P: Conjugated linoleic acid improves blood pressure by increasing adiponectin and endothelial nitric oxide synthase activity. $J$ Nutr Biochem 23: 487-493, 2012a.

DECLERCQ V, TAYLOR CG, ZAHRADKA P: Isomer-specific effects of conjugated linoleic acid on blood pressure, adipocyte size and function. Br J Nutr 107: 1413-1421, 2012b.

DERAKHSHANDE-RISHEHRI SM, MANSOURIAN M, KELISHADI R, HEIDARI-BENI M: Association of foods enriched in conjugated linoleic acid (CLA) and CLA supplements with lipid profile in human studies: a systematic review and meta-analysis. Public Health Nutr 18: 2041-2054, 2015.

DILZER A, PARK Y: Implication of conjugated linoleic acid (CLA) in human health. Crit Rev Food Sci Nutr 52: 488-513, 2012.

DIXON JL, STOOPS JD, PARKER JL, LAUGHLIN MH, WEISMAN GA, STUREK M: Dyslipidemia and vascular dysfunction in diabetic pigs fed an atherogenic diet. Arterioscler Thromb Vasc Biol 19: 2981-2992, 1999.

GARCÍA-PRIETO CF, HERNÁNDEZ-NUÑO F, RIO DD, RUIZ-HURTADO G, ARÁNGUEZ I, RUIZ-GAYO M, SOMOZA B, FERNÁNDEZ-ALFONSO MS: High-fat diet induces endothelial dysfunction through a downregulation of the endothelial AMPK-PI3K-Akt-eNOS pathway. Mol Nutr Food Res 59: 520-532, 2015.

HADI HA, CARR CS, AL SUWAIDI J: Endothelial dysfunction: cardiovascular risk factors, therapy, and outcome. Vasc Health Risk Manag 1: 183-198, 2005.

HAYEK T, ITO Y, AZROLAN N, VERDERY RB, AALTO-SETÄLÄ K, WALSH A, BRESLOW JL: Dietary fat increases high density lipoprotein (HDL) levels both by increasing the transport rates and decreasing the fractional catabolic rates of HDL cholesterol ester and apolipoprotein (Apo) A-I. Presentation of a new animal model and mechanistic stud. J Clin Invest 91: 1665-1671, 1993.

HERMANS MP, AHN SA, ROUSSEAU MF: The non-HDL-C/HDL-C ratio provides cardiovascular risk stratification similar to the ApoB/ApoA1 ratio in diabetics: comparison with reference lipid markers. Diabetes Metab Syndr Clin Res Rev 1: 23-28, 2007.

HIATT WR, KAUL S, SMITH RJ: The cardiovascular safety of diabetes drugs--insights from the rosiglitazone experience. $N$ Engl J Med 369: 1287-1289, 2013.

HUANG BW, CHIANG MT, YAO HT, CHIANG W: The effect of high-fat and high-fructose diets on glucose tolerance and plasma lipid and leptin levels in rats. Diabetes Obes Metab 6: 120-126, 2004. 
IM M, KIM M, LEE JK, CHANG YH, LEE DY, HONG S-I, LEE YY, HONG YJ: HDL cholesterol reduction during rosiglitazone and fenofibrate treatment in a type 2 diabetes mellitus patient with dyslipidemia (in Korean). Korean J Lab Med 30: 17-19, 2010.

ISENBERG JS, MARTIN-MANSO G, MAXHIMER JB, ROBERTS DD: Regulation of nitric oxide signalling by thrombospondin 1: implications for anti-angiogenic therapies. Nat Rev Cancer 9: 182-194, 2009.

KAGOTA S, TANAKA N, KUBOTA Y, YAMAGUCHI Y, NAKAMURA K, KUNITOMO M, SHINOZUKA K: Characteristics of vasorelaxation responses in a rat model of metabolic syndrome. Clin Exp Pharmacol Physiol 31 (Suppl 2): S54-S56, 2004.

KAUR J: A comprehensive review on metabolic syndrome. Cardiol Res Pract 2014: 943162, 2014.

KIM SW, JEE JH, KIM HJ, JIN SM, SUH S, BAE JC, KIM SW, CHUNG JH, MIN YK, LEE MS, LEE MK, KIM KW, KIM JH: Non-HDL-cholesterol/HDL-cholesterol is a better predictor of metabolic syndrome and insulin resistance than apolipoprotein B/apolipoprotein A1. Int J Cardiol 168: 2678-2683, 2013.

KLEINHENZ JM, MURPHY TC, POKUTTA-PASKALEVA AP, GLEASON RL, LYLE AN, TAYLOR WR, BLOUNT MA, CHENG J, YANG Q, SUTLIFF RL, HART CM: Smooth muscle-targeted overexpression of peroxisome proliferator activated receptor- $\gamma$ disrupts vascular wall structure and function. PLoS One 10: 1-25, 2015.

LESSARD SJ, RIVAS DA, CHEN ZP, BONEN A, FEBBRAIO MA, REEDER DW, KEMP BE, YASPELKIS BB, HAWLEY JA: Tissue-specific effects of rosiglitazone and exercise in the treatment of lipid-induced insulin resistance. Diabetes 56: 1856-1864, 2007.

LINZ PE, LOVATO LC, BYINGTON RP, O’CONNOR PJ, LEITER LA, WEISS D, FORCE RW, CROUSE JR, ISMAIL-BEIGI F, SIMMONS DL, PAPADEMETRIOU V, GINSBERG HN, ELAM MB: Paradoxical reduction in HDL-C with fenofibrate and thiazolidinedione therapy in type 2 diabetes: the ACCORD lipid trial. Diabetes Care 37: 686-693, 2014.

LIU LF, PURUSHOTHAM A, WENDEL AA, BELURY MA: Combined effects of rosiglitazone and conjugated linoleic acid on adiposity, insulin sensitivity, and hepatic steatosis in high-fat-fed mice. Am J Physiol Gastrointest Liver Physiol 292: G1671-G1682, 2007.

LOONG BJ, TAN JH, LIM KH, MBAKI Y, TING KN: Contractile function of smooth muscle retained after overnight storage. Naunyn Schmiedebergs Arch Pharmacol 388: 1061-1067, 2015.

LU W, RESNICK HE, JABLONSKI KA, JONES KL, JAIN AK, HOWARD WJ, ROBBINS DC, HOWARD BV: Non-HDL cholesterol as a predictor of cardiovascular disease in type 2 diabetes: the strong heart study. Diabetes Care 26: 16-23, 2003.

MARTIROSYAN DM, MIROSHNICHENKO LA, KULAKOVA SN, POGOJEVA AV, ZOLOEDOV VI: Amaranth oil application for coronary heart disease and hypertension. Lipids Health Dis 6: 1, 2007.

MOHANKUMAR SK, ZAHRADKA P, TAYLOR C: Does conjugated linoleic acid boost or battle metabolic syndrome? Clin Lipidol 6: 501-505, 2011.

MOHANKUMAR SK, HANKE D, SIEMENS L, CATTINI A, ENNS J, SHEN J, REANEY M, ZAHRADKA P, TAYLOR CG: Dietary supplementation of trans-11-vaccenic acid reduces adipocyte size but neither aggravates nor attenuates obesity-mediated metabolic abnormalities in fa/fa Zucker rats. Br $J$ Nutr 109: 1628-1636, 2013.

MOORADIAN A: Dyslipidemia in type 2 diabetes mellitus. Nat Clin Pract Endocrinol Metab 5: 150-159, 2009.

MOYA-CAMARENA SY, VANDEN HEUVEL JP, BLANCHARD SG, LEESNITZER LA, BELURY MA: Conjugated linoleic acid is a potent naturally occurring ligand and activator of PPARalpha. J Lipid Res 40: 1426-1433, 1999.

MURTHY SN, OBREGON DF, CHATTERGOON NN, FONSECA NA, MONDAL D, DUNNE JB, DIEZ JG, JETER JR JR, KADOWITZ PJ, AGRAWAL KC, MCNAMARA DB, FONSECA VA: Rosiglitazone reduces serum homocysteine levels, smooth muscle proliferation, and intimal hyperplasia in Sprague-Dawley rats fed a high methionine diet. Metabolism 54: 645-652, 2005.

NISSEN SE, WOLSKI K: Effect of rosiglitazone on the risk of myocardial infarction and death from cardiovascular causes. N Engl J Med 356: 2457-2471, 2007. 
NISSEN SE, WOLSKI K: Rosiglitazone revisited: an updated meta-analysis of risk for myocardial infarction and cardiovascular mortality. Arch Intern Med 170: 1191-1201, 2010.

NOONE EJ, ROCHE HM, NUGENT AP, GIBNEY MJ: The effect of dietary supplementation using isomeric blends of conjugated linoleic acid on lipid metabolism in healthy human subjects. Br J Nutr 88: 243-251, 2002.

NORMEN L, FROHLICH J, MONTANER J, HARRIS M, ELLIOTT T, BONDY G: Combination therapy with fenofibrate and rosiglitazone paradoxically lowers serum HDL cholesterol. Diabetes Care 27: 2241-2242, 2004.

NOTO A, ZAHRADKA P, YURKOVA N, XIE X, NITSCHMANN E, OGBORN M, TAYLOR CG: Conjugated linoleic acid reduces hepatic steatosis, improves liver function, and favorably modifies lipid metabolism in obese insulin-resistant rats. Lipids 41: 179-188, 2006.

ONUTA G, HILLEBRANDS JL, RIENSTRA H, BOER MW, KLATTER FA, NAVIS G, ROZING J, ROKS AJ: Dichotomous effects of rosiglitazone in transplantation-induced systemic vasodilator dysfunction in rats. Transplantation 85: 582-588, 2008.

PARIZA MW, PARK Y, COOK ME: Conjugated linoleic acid and the control of cancer and obesity. Toxicol Sci 52: 107-110, 1999.

PRANPRAWIT A, WOLBER FM, HEYES JA, MOLAN AL, KRUGER MC: Short-term and long-term effects of excessive consumption of saturated fats and/or sucrose on metabolic variables in Sprague Dawley rats: a pilot study. J Sci Food Agric 93: 3191-3197, 2013.

SCHWING W, HUSTAK L, TAYLOR H: Paradoxical severe decrease in high-density lipoprotein cholesterol due to rosiglitazone-fenofibrate interaction. Endocr Pract 16: 382-388, 2010.

SENA CM, PEREIRA AM, SEIÇA R: Endothelial dysfunction - a major mediator of diabetic vascular disease. Biochim Biophys Acta 1832: 2216-2231, 2013.

SHOKRYZADAN P, RAJION MA, MENG GY, BOO LJ, EBRAHIMI M, ROYAN M, SAHEBI M, AZIZI P, ABIRI R, JAHROMI MF: Conjugated linoleic acid: a potent fatty acid linked to animal and human health. Crit Rev Food Sci Nutr 57: 2737-2748, 2017.

SOARES AG, DE CARVALHO MHC, AKAMINE E: Obesity induces artery-specific alterations: evaluation of vascular function and inflammatory and smooth muscle phenotypic markers. Biomed Res Int 2017: 5038602, 2017.

STAPLETON PA, JAMES ME, GOODWILL AG, FRISBEE JC: Obesity and vascular dysfunction. Pathophysiology 15: 79-89, 2008.

STARK AH, TIMAR B, MADAR Z: Adaptation of Sprague Dawley rats to long-term feeding of high fat or high fructose diets. Eur J Nutr 39: 229-234, 2000.

TAYLOR CG, ZAHRADKA P: Dietary conjugated linoleic acid and insulin sensitivity and resistance in rodent models. Am J Clin Nutr 79 (6 Suppl): 1164S-1168S, 2004.

UMRANI DN, BANDAY AA, HUSSAIN T, LOKHANDWALA MF: Rosiglitazone treatment restores renal dopamine receptor function in obese Zucker rats. Hypertension 40: 880-885, 2002.

VAN DEVENTER HE, MILLER WG, MYERS GL, SAKURABAYASHI I, BACHMANN LM, CAUDILL SP, DZIEKONSKI A, EDWARDS S, KIMBERLY MM, KORZUN WJ, LEARY ET, NAKAJIMA K, NAKAMURA M, SHAMBUREK RD, VETROVEC GW, WARNICK GR, REMALEY AT: Non-HDL cholesterol shows improved accuracy for cardiovascular risk score classification compared to direct or calculated LDL cholesterol in a dyslipidemic population. Clin Chem 57: 490-501, 2011.

WANDERS AJ, BROUWER IA, SIEBELINK E, KATAN MB: Effect of a high intake of conjugated linoleic acid on lipoprotein levels in healthy human subjects. PLoS One 5: e9000, 2010.

WATTS SW, MCCLAIN JL, DORRANCE AM: Perivascular fat impairs contraction in aorta from obese but not lean adult rats. FASEB J 26 (Suppl 1): 1115.4, 2012.

XU ZJ, FAN JG, DING XD, QIAO L, WANG GL: Characterization of high-fat, diet-induced, non-alcoholic steatohepatitis with fibrosis in rats. Dig Dis Sci 55: 931-940, 2010.

ZHAO D, MCCULLY BH, BROOKS VL: Rosiglitazone improves insulin sensitivity and baroreflex gain in rats with diet-induced obesity. J Pharmacol Exp Ther 343: 206-213, 2012. 